\title{
Animals in research-where to draw the line.
}

\author{
Abdul Gafoor Puthiyaveetil* \\ Department of Biotechnology, American University of Ras Al Khaimah, UAE
}

\begin{abstract}
Animal research is vital for the advancement of science, particularly in the field of biomedical research. However; due to ethical concerns, each researcher needs to take the responsibility for animal research and ensure that the use of animals in their research is justified.
\end{abstract}

Keywords: Animal research, immune disorders.

\section{Editorial}

Over the past few decades, the biomedical research has seen a tremendous growth in terms of medicines and treatment strategies against various disease conditions. This is mostly attributed by the success of well-planned research using animal models. Commonly used animals include fishes, birds, mice, rats, rabbits, cats, dogs and primates. In many cases, these animals are genetically modified to reflect a certain disease condition in humans or treated with certain drugs in their initial stages of drug development. However; as the animals move up in the sentient hierarchy list, the number of people opposing the animal research increases proportionately. This has led to different conflicts in research and a need for careful planning is sought when doing animal experiments.

Different animals are currently used as model organisms for a wide variety of diseases, including cancer, organ specific diseases, immune disorders, aging studies, infectious diseases, and life-style diseases. In addition, they are used in physiological studies such as nutritional and hormonal studies. The use of animal models in biomedical research in unquestionable, owing to the tremendous progress we achieved in the past few decades.

Even with all these advances, it is generally accepted that many of the genetically modified animals serve a limited purpose in biomedical research. It is not uncommon to have embryonic/ fetal mortality or serious birth defects in the animal model. Many animal models show significant variations from humans in their physiological and pathological mechanisms of diseases and so, the results cannot be translated directly. Recent studies suggest that the strain of animals play a crucial role in the results, and a majority of results are applicable only to the specific strain. When animals are bred in captivity for research, many unwanted animals are generated, which will be promptly culled after their initial validation.

Animal rights organizations and a considerable fraction of the general public have expressed serious concerns regarding the use of animals in biomedical research. In light of these, different regulatory bodies have delineated rules and guidelines on conducting animal research in order to streamline the experiments, and to avoid unnecessary pain for the animals. A generally accepted rule, 3Rs, advocate the replacement, reduction and refinement of animal research. Replacing the animal research with simulations or cell in vitro studies is impossible in many cases. A proper statistical analysis for conducting research and a well-planned breeding program can reduce the number of animals significantly. The last $\mathrm{R}$, refinement, asks for measures to reduce unwanted pain and suffering for the animals. This is widely practiced in most of the certified laboratories by using anesthetics, modified procedures, and better living environments for the animals.

The current regulations are enforced by the researcher's institution itself and the standards can vary widely based on the interpretation of policies by each institution. When it comes to the actual research, it is ultimately up to each researcher to ensure that the proposed animal studies are necessary for the advancement of science. Sufficient research, statistical analysis, and proper planning can reduce repetition of animal experiments, lower animal numbers, and reduce animal sufferings in biomedical research. 